\title{
The role of the gut microbiota on the metabolic status of obese children
}

\author{
Xin Yuan ${ }^{1}$, Ruimin Chen ${ }^{1 *}$, Kenneth L. McCormick ${ }^{2}$, Ying Zhang ${ }^{1}$, Xiangquan Lin ${ }^{1}$ and Xiaohong Yang ${ }^{1}$
}

\begin{abstract}
Background: The term "metabolically healthy obese (MHO)" denotes a hale and salutary status, yet this connotation has not been validated in children, and may, in fact, be a misnomer. As pertains to obesity, the gut microbiota has garnered attention as conceivably a nosogenic or, on the other hand, protective participator.

Objective: This study explored the characteristics of the fecal microbiota of obese Chinese children and adolescents of disparate metabolic statuses, and the associations between their gut microbiota and circulating proinflammatory factors, such as IL-6, TNF-a, lipopolysaccharide-binding protein (LBP), and a cytokine up-regulator and mediator, leptin.
\end{abstract}

Results: Based on weight and metabolic status, the 86 Chinese children (ages 5-15 years) were divided into three groups: metabolically healthy obese $(\mathrm{MHO}, \mathrm{n}=42)$, metabolic unhealthy obese $(\mathrm{MUO}, \mathrm{n}=23)$, and healthy normal weight controls (Con, $\mathrm{n}=21$ ). In the MUO subjects, the phylum Tenericutes, as well as the alpha and beta diversity, were significantly reduced compared with the controls. Furthermore, Phylum Synergistetes and genus Bacteroides were more prevalent in the MHO population compared with controls. For the MHO group, Spearman's correlation analysis revealed that serum IL-6 positively correlated with genus Paraprevotella, LBP was positively correlated with genus Roseburia and Faecalibacterium, and negatively correlated with genus Lactobacillus, and leptin correlated positively with genus Phascolarctobacterium and negatively with genus Dialister (all $p<0.05$ ).

Conclusion: Although there are distinct differences in the characteristic gut microbiota of the MUO population versus $\mathrm{MHO}$, dysbiosis of gut microsystem is already extant in the $\mathrm{MHO}$ cohort. The abundance of some metabolismrelated bacteria associates with the degree of circulating inflammatory compounds, suggesting that dysbiosis of gut microbiota, present in the $\mathrm{MHO}$ children, conceivably serves as a compensatory or remedial response to a surfeit of nutrients.

Keywords: Metabolically healthy obese, Children, 16s rRNA, Gut microbiota

\section{Introduction}

The global epidemic of childhood obesity, and the accompanying rise in the prevalence of endocrine, metabolic, and cardiovascular comorbidities, is perhaps the most impactful and ubiquitous public health disorder of the modern world [1]. In the context of this pandemic, a

*Correspondence: chenrm321@163.com

${ }^{1}$ Department of Endocrinology, Fuzhou Children's Hospital of Fujian Medical University, NO. 145, 817 Middle Road, Fuzhou 350005, China

Full list of author information is available at the end of the article distinct group of youth with obesity who are devoid of metabolic disturbances-so-called "metabolically healthy obese" (MHO)- have been identified. Obesity notwithstanding, by definition $\mathrm{MHO}$ children retain a favorable metabolic profile, with preserved insulin sensitivity along with normal blood pressure, glucose homeostasis, lipids, and liver enzymes. Moreover, their hormonal, inflammation, and immune profiles are seemingly impervious to obesity [2]. First described in obese adults, the MHO phenotype has also been extensively studied in young people with obesity [2]. Arguably, MHO may be 
a transitional stage to the far more common, more highrisk, conventional cardio-metabolic obese phenotype. Regardless of the aforesaid normal biochemical characteristics of $\mathrm{MHO}$, the risk for cardiovascular disease persists since the MHO phenotype may be unstable, thereby transitory [3, 4].

Among the non-genetic factors associated with obesity, the gut microbiota has garnered attention as an obesity regulator given the robust correlations in animal studies between gut microbiota and body weight. Obese individuals, whether adults or children, have increased abundance in Firmicutes in concert with decreased in Bacteroidetes [5, 6]. The distinctive gut microbiota prevalent in obese subjects is recognized as promoting an unhealthy metabolic obese (MUO) phenotype with attendant comorbidities, such as increased endotoxemia, intestinal and systemic inflammation, as well as insulin resistance. An altered gut microbiota has been implicated in obesity and type 2 diabetes mellitus (T2DM) insofar as a decrement in certain species and gene richness have been linked to adiposity, dyslipidemia, and insulin resistance [7]. Hence, the clinical repercussions aside, it is plausible that differences in the gut microbiota could dictate whether an obese child is metabolically fit (MHO) or not (MUO) $[8,9]$.

Obesity and related metabolic disorders are associated with gut microbiota dysbiosis, disrupted intestinal barrier and chronic inflammation [10]. For instance, obese Mexican children and adolescents had increased levels of leptin and C-reactive protein, which were associated with changes in the gut microbiota [11]. However, the association between gut microbiota and proinflammatory cytokines, such as IL-6, TNF- $\alpha$ and lipopolysaccharidebinding protein (LBP), has not been fully investigated in children of varying metabolic statues. Firstly, this study examined the metabolic heterogeneity of obese children as it relates to the composition of the gut microbiota. And, as a secondary end point, identify metabolic-specific bacteria which associate with serum inflammatory factors incriminated in obesity comorbidities.

\section{Results}

\section{Study participants}

Based on weight status, the metabolically stable cohort subjects $(n=63)$ were subdivided as MHO $(n=42)$ or Con $(n=21)$.

The age of the 86 participates ranged from 5.5 to 14.3 years, with a mean of $9.76 \pm 1.93$ years. There were 65 obese children, of whom 23 were MUO and 42 were MHO. The BMI of other 21 children were normal. Age, weight, BMI, BMI-Z, WHtR, SBP, TG and LDL-c in the MUO group were significantly higher than the Con and MHO children, and HDL-c in the in the MUO group were significantly lower than the Con and MHO children (all $\mathrm{p}<0.05$, Table 1 ).

The weight, BMI, BMI-Z, WHR, WHtR, SBP, DBP, TG, LDL-c, IL-6, TNF- $\alpha$, LBP and leptin were significantly higher in the MHO group than the Con children, and HDL-c in the MHO group were significantly lower than the Con group (all $\mathrm{p}<0.05$ ). There was no statistical difference in age, gender, FPG and fasting TC between $\mathrm{MHO}$ and Con (all p >0.05, Table 1).

\section{Microbiota profiles in different metabolic status subjects}

A total of 918,578 sequencing reads were obtained from 86 fecal samples, with an average value of 10,681 counts per sample. We identified an overall of 146 OTUs, among which 136 OTU with $\geq 2$ counts, and they were grouped in 9 phylum and 38 families.

\section{Abundance profiling in different metabolic status subjects} Grouping OTUs at phylum level, and applying the Mann-Whitney $U$ test on the relative abundances of phyla for the two groups, the relative abundances of phylum Tenericutes was more prevalent in the metabolically healthy cohorts (Con and MHO children) compared to the MUO group $(\mathrm{p}=0.006$, Additional file 1: Table S1 and Fig. 1a).

On OTUs at the genera level, by Mann-Whitney U-test, including all the genera (merging small taxa with counts $<10$ ), we identified that genera Anaerostipes, Alistipes, Desulfovibrio, Fusobacterium, Gemmiger, Odoribacter, Oscillospira and Parabacteroides were more prevalent in the metabolically healthy cohorts (Con and MHO children) versus MUO children, yet the genus Dorea was more prevalent in MUO $(p<0.05$; Fig. $1 \mathrm{~b}$, Table 2).

\section{Alpha- and beta-diversity in different metabolic status subjects}

To assess the overall differences of microbial community structures in metabolic healthy and MUO subjects, we measured ecological parameters based on alpha-diversity. The alpha-diversity analysis showed significantly higher diversity in metabolic healthy subjects (Con and MHO children) in comparison to MUO participants ( $\mathrm{p}<0.05$, Fig. 2a, b, Additional file 1: Table S2).

To determine the differences between microbial community profiles in metabolic healthy and MUO subjects, we calculated beta-diversity. By Distance method BrayCurtis dissimilarities PCoA analysis, the gut microbiota samples from Con and $\mathrm{MHO}$ children were clustered together and separated partly from the MUO group. Upon analysis, the first coordinate (Axis.1) explained the $18.6 \%$ of the inter sample variance the second coordinate (Axis.2) explained the $14.5 \%$ of the inter sample variance 
Table 1 Anthropometric profiles and laboratory measurements

\begin{tabular}{|c|c|c|c|c|}
\hline & \multirow[t]{2}{*}{ MUO $(n=23)$} & \multicolumn{3}{|c|}{ Metabolic healthy subjects } \\
\hline & & Total $(n=63)$ & $\mathrm{MHO}(\mathrm{n}=42)$ & $\operatorname{Con}(n=21)$ \\
\hline Age (year) & $10.96 \pm 1.69$ & $9.32 \pm 1.84^{*}$ & $9.47 \pm 1.68^{*}$ & $9.02 \pm 2.14$ \\
\hline Male (\%) & 65.2 & 50.8 & 54.8 & 42.9 \\
\hline Weight (kg) & $61.4 \pm 11.5$ & $43.0 \pm 14.6^{*}$ & $49.6 \pm 12.4^{*}$ & $29.9 \pm 8.5^{\#}$ \\
\hline $\mathrm{BMI}\left(\mathrm{kg} / \mathrm{m}^{2}\right)$ & $27.02 \pm 2.75$ & $21.80 \pm 4.91^{*}$ & $24.65 \pm 3.14^{*}$ & $16.11 \pm 1.91^{\#}$ \\
\hline BMI-Z & $2.81 \pm 0.61$ & $1.77 \pm 1.53^{*}$ & $2.74 \pm 0.60$ & $-0.16 \pm 0.79^{\#}$ \\
\hline WHR & $0.89 \pm 0.05$ & $0.86 \pm 0.06$ & $0.88 \pm 0.05$ & $0.84 \pm 0.06^{\#}$ \\
\hline WHtR & $0.55 \pm 0.04$ & $0.50 \pm 0.06^{*}$ & $0.53 \pm 0.04$ & $0.43 \pm 0.03^{\#}$ \\
\hline $\mathrm{SBP}(\mathrm{mmHg})$ & $116.45 \pm 8.77$ & $101.52 \pm 8.36^{*}$ & $105.51 \pm 6.96^{*}$ & $94.48 \pm 5.51^{\#}$ \\
\hline $\mathrm{DBP}(\mathrm{mmHg})$ & $65.09 \pm 5.72$ & $62.57 \pm 5.79$ & $63.81 \pm 6.45$ & $60.38 \pm 3.56 \#$ \\
\hline FPG (mmol/L) & $5.09 \pm 0.67$ & $4.87 \pm 0.39$ & $4.82 \pm 0.38^{*}$ & $4.97 \pm 0.40$ \\
\hline $\mathrm{TC}(\mathrm{mmol} / \mathrm{L})$ & $4.54 \pm 0.90$ & $4.30 \pm 0.62$ & $4.39 \pm 0.57$ & $4.14 \pm 0.69$ \\
\hline $\mathrm{TG}(\mathrm{mmol} / \mathrm{L})$ & $1.62 \pm 0.99$ & $0.86 \pm 0.30^{*}$ & $0.93 \pm 0.33^{*}$ & $0.72 \pm 0.19^{\#}$ \\
\hline LDL-c (mmol/L) & $2.65 \pm 0.66$ & $2.31 \pm 0.53^{*}$ & $2.45 \pm 0.48$ & $2.03 \pm 0.54 \#$ \\
\hline $\mathrm{HDL}-\mathrm{c}(\mathrm{mmol} / \mathrm{L})$ & $1.24 \pm 0.24$ & $1.58 \pm 0.30^{*}$ & $1.51 \pm 0.30^{*}$ & $1.71 \pm 0.26^{\#}$ \\
\hline Leptin ( $\mu \mathrm{g} / \mathrm{mL})$ & $2.70 \pm 1.48$ & $2.23 \pm 1.83$ & $3.10 \pm 1.65$ & $0.51 \pm 0.35^{* \#}$ \\
\hline TNF-a (pg/mL) & $47.50 \pm 25.63$ & $48.48 \pm 18.77$ & $53.43 \pm 17.88$ & $38.59 \pm 16.81^{\#}$ \\
\hline $\mathrm{IL}-6(\mu \mathrm{g} / \mathrm{mL})$ & $1.76 \pm 0.86$ & $1.65 \pm 0.93$ & $1.86 \pm 1.04$ & $1.23 \pm 0.42^{* \#}$ \\
\hline $\operatorname{LBP}(\mu \mathrm{g} / \mathrm{mL})$ & $34.8(29.55,41.20)$ & $33.66(27.01,38.95)$ & $33.28(27.75,41.22)$ & $27.18(22.02,36.61)^{* \#}$ \\
\hline
\end{tabular}

in metabolic healthy subjects (Con and MHO children) in comparison to MUO participants $(\mathrm{P}=0.038$, Fig. 2e, Additional file 1: Table S3).

\section{Bacterial taxa differences in different metabolic status subjects}

We next used LEfSe analysis to identify bacteria in which the relative abundance was significantly increased or decreased in each phenotypic category. The Con and MHO children had members of the phylum Tenericutes, class Deltaproteobacteria, Mollicutes, order Desulfovibrionales, RF39, family Christensenellaceae, Odoribacteraceae, Porphyromonadaceae, Ruminococcaceae, genera Anaerostipes, Oscillospira, Odoribacter, Gemmiger, Parabacteroides, Alistipes, that were significantly higher than MUO subjects. Furthermore, the MUO subjects had members of the genus Fusobacterium that were significantly higher than the Con and MHO children (all $\mathrm{p}<0.05$, Fig. 3a, b).

\section{Microbiota profiles in obese children with different metabolic status \\ Abundance profiling}

Grouping OTUs at phylum level, and applying the Mann-Whitney $U$ test on the relative abundances of phyla for the MHO and MUO groups, the relative abundance of phylum Tenericutes was more prevalent in the $\mathrm{MHO}$ group compared to the MUO group $(\mathrm{p}=0.027$, Table 3 and Fig. 1c).

On OTUs at the genera level, by Mann-Whitney U analysis, including all the genera (merging small taxa with counts $<10$ ), we identified that genera Desulfovibrio, Parabacteroides and Gemmiger were more prevalent in MHO subjects compared to MUO subjects ( $p=0.027$, 0.040 and 0.047 , respectively; Fig. 1d).

\section{Alpha- and beta-diversity between $\mathrm{MHO}$ and MUO subjects}

Regarding alpha-diversity, in both the MHO and MUO group, the analysis exposed significantly higher diversity in $\mathrm{MHO}$ subjects versus MUO participants (all $\mathrm{p}<0.05$, Fig. 2c, d, Additional file 1: Table S2).

Regarding beta-diversity, by an unweighted-UniFrac method, the MHO group was lower than the MUO group ( $p=0.021$, Additional file 1: Table S3).

\section{Bacterial taxa differences between MHO and MUO subjects} LEfSe analysis showed MHO subjects had members of the phylum Tenericutes, class Deltaproteobacteria, Mollicutes, order Desulfovibrionales, RF39, family Christensenellaceae, Odoribacteraceae, Rikenellaceae, 
$\mathbf{a}$

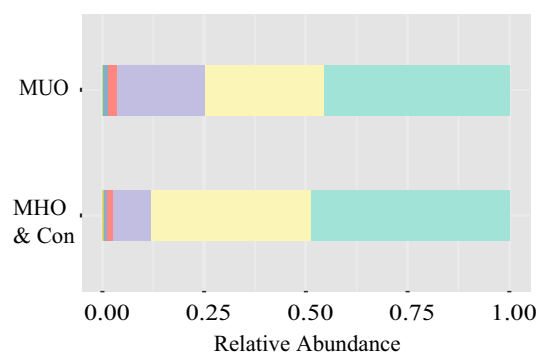
Phylum $\begin{array}{ll}\text { Firmicutes } \square \text { Actinobacteria } \square \text { Verrucomicrobia } \\ \text { Bacteroidetes } \square \text { Fusobacteria } \square 7\end{array}$ - Proteobacteria 1 Tenericutes Synergistetes

c

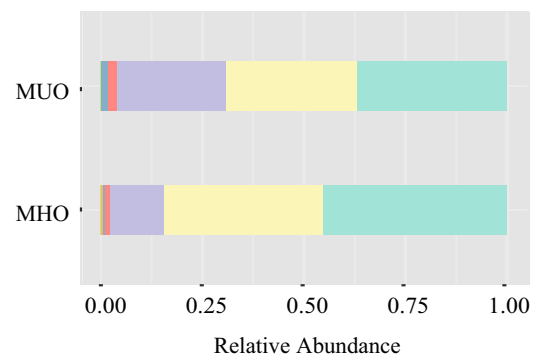

Phylum $\begin{aligned} & \text { Bacteroidetes } \\ & \text { Firmicutes }\end{aligned}$ Proteobacteria Tenericutes

$\mathbf{e}$

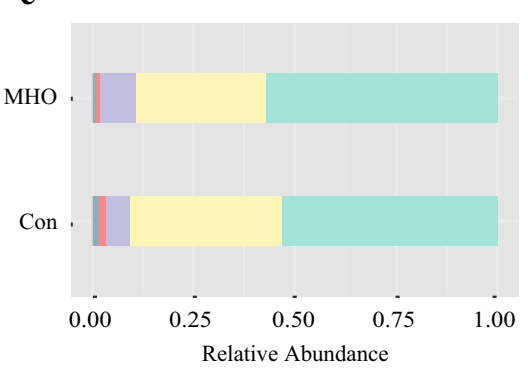
$\begin{array}{lll} & \text { Firmicutes } & \text { Fusobacteria } \\ \text { Bacteroidetes } & \text { Synergistetes } \\ \text { Phylum } & \text { Tenicutes } \\ \text { Proteobacteria } & \text { Verrucomicrobia }\end{array}$ b

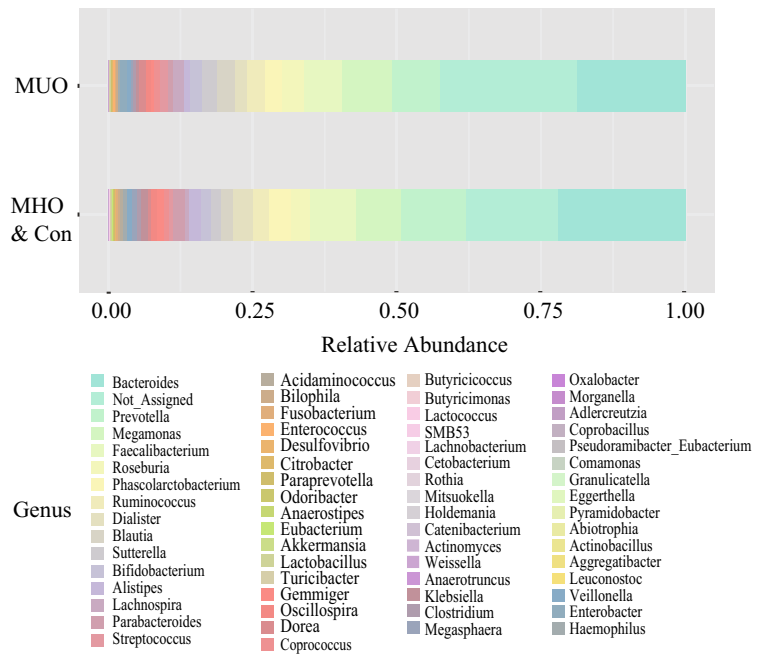

d

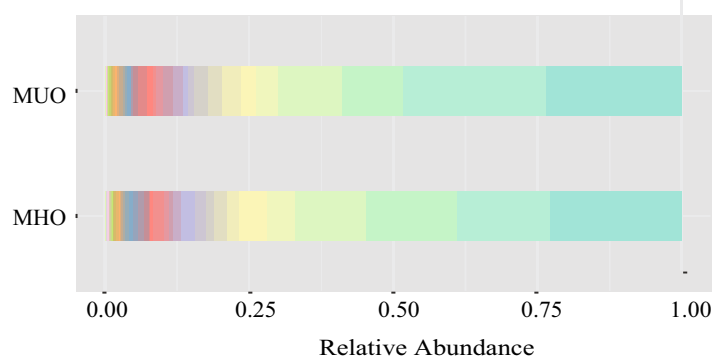

f

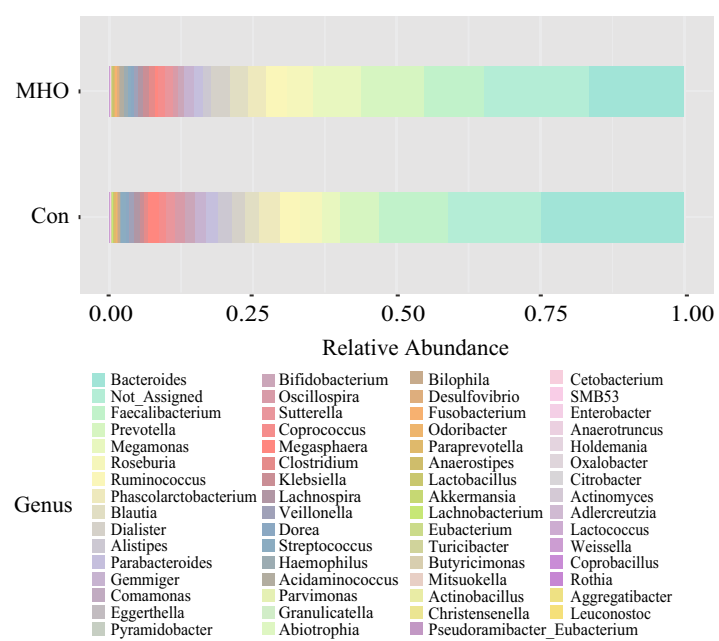

Fig. 1 Bar chart representing Mann-Whitney U-test results on operational taxonomic units (OTUs) grouped in phyla (a, c, e) and in genus (b, d, f) of the different metabolic status groups. Each column in the plot represents a group, and each color in the column represents the percentage of relative abundance for each OTU. MUO metabolic unhealthy obese, $M H O$ metabolically healthy obese, Con controls 
Table 2 The mean relative abundance of gut microbiota with significantly differences in different metabolic status at genera level

\begin{tabular}{lllll}
\hline & MUO & MHO and Con & Z & P value \\
\hline Anaerostipes & 0.001 & 0.001 & -2.084 & 0.037 \\
Odoribacter & 0.000 & 0.002 & -2.122 & 0.034 \\
Desulfovibrio & 0.000 & 0.003 & -2.142 & 0.032 \\
Alistipes & 0.010 & 0.023 & -2.182 & 0.029 \\
Fusobacterium & 0.001 & 0.002 & -2.185 & 0.029 \\
Dorea & 0.012 & 0.005 & -2.288 & 0.022 \\
Gemmiger & 0.007 & 0.013 & -2.320 & 0.020 \\
Oscillospira & 0.008 & 0.010 & -2.445 & 0.014 \\
Parabacteroides & 0.007 & 0.020 & -2.552 & 0.011 \\
\hline
\end{tabular}

MUO metabolic unhealthy obese, $\mathrm{MHO}$ metabolically healthy obese, Con controls

Desulfovibrionaceae, Porphyromonadaceae, Ruminococcaceae, genus Gemmiger, Parabacteroides that were significantly higher than MUO subjects (all $\mathrm{p}<0.05$, Fig. 3c, d).

\section{Microbiota profiles in $\mathrm{MHO}$ and Con children with different weight status \\ Abundance profiling}

Grouping OTUs at phylum level, the relative abundances of phylum Synergistetes was more prevalent in the MHO group compared to the Con group ( $<<0.05$, Fig. 1e, Table 4).

On OTUs at the genera level, including all the genera (merging small taxa with counts $<10$ ), genera Anaerotruncus, Bacteroides, Adlercreutzia and Pyramidobacter were more prevalent in MHO subjects versus MUO subjects $(p<0.05$; Fig. 1f).

\section{Alpha- and beta-diversity between different weight status}

Regarding alpha-diversity, the Shannon diversity index, Observed OTUs, Faith's phylogenetic diversity and Pielou's evenness based on OTU distribution did not reveal any significant difference between $\mathrm{MHO}$ and Con (all $\mathrm{p}>0.05$, Additional file 1: Table S2); also, beta-diversity did not differ significantly between these two groups. Importantly, none of the comparisons were significantly different (all $\mathrm{p}>0.05$ ) after correction for multiple testing (Additional file 1: Table S3).
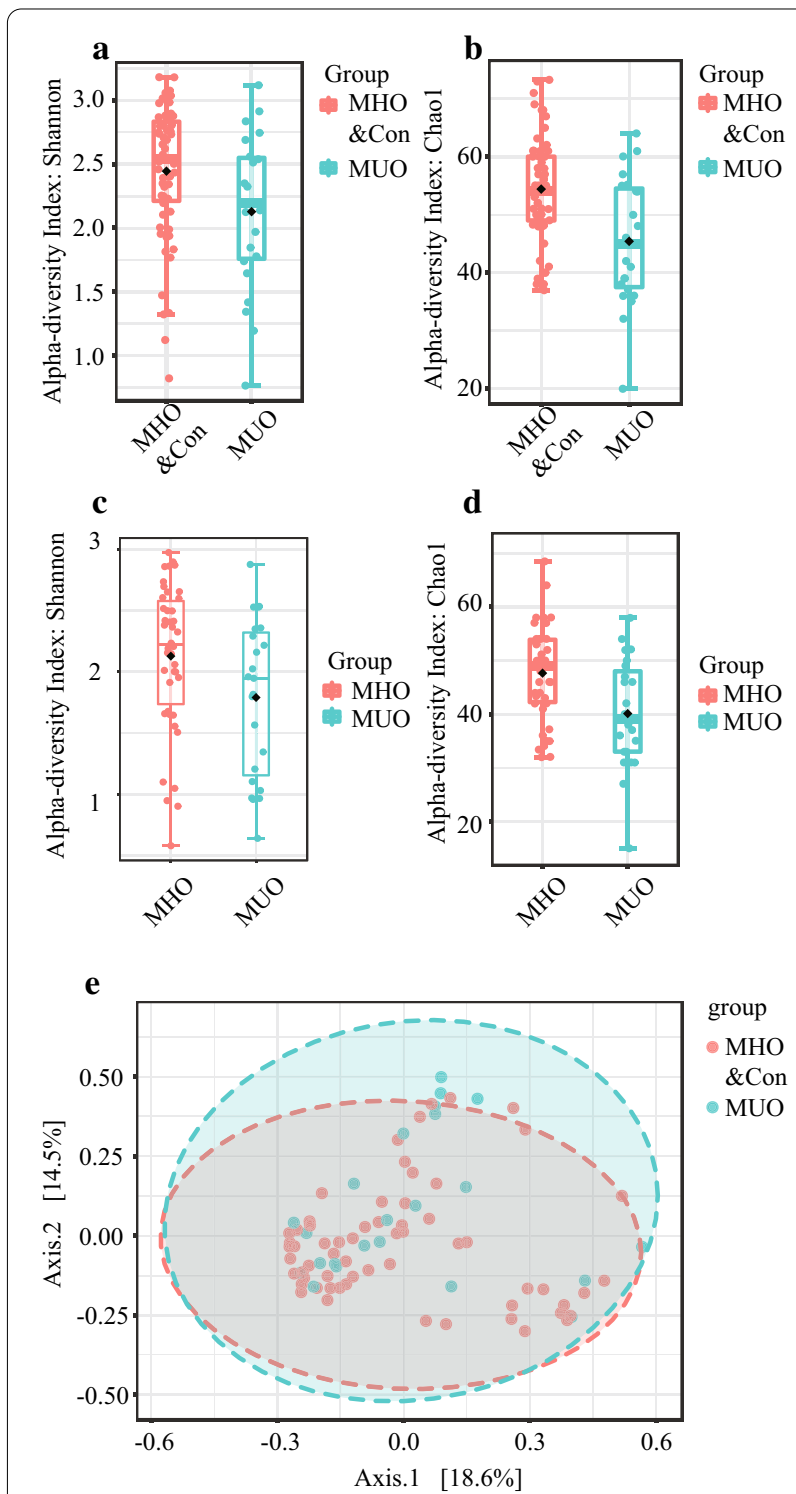

Fig. 2 Characterization of alpha- and beta-diversity of the gut microbiota in Con, $\mathrm{MUO}$ and $\mathrm{MHO}$ groups. The $\mathrm{y}$-axes show the Shannon index $(\mathbf{a}, \mathbf{c})$ and Chao 1 richness index $(\mathbf{b}, \mathbf{d})$. The $\mathrm{x}$-axes show the phenotypic categories. Additional data are in Additional file 1: Table S2. Principal coordinates analysis (PCOA) plot of Con and MHO children and MUO subjects (e). The plots show the first two principal coordinates (axes) for PCoA using Bray-Curtis Distance method. MUO metabolic unhealthy obese, $\mathrm{MHO}$ metabolically healthy obese, Con controls

\section{Bacterial taxa differences in $\mathrm{MHO}$ and Con children of different weight status}

LEfSe analysis showed MHO subjects had members of the phylum Synergistetes, class Synergistia, order Synergistales, Erysipetotrichales, family Dethiosulfovibrionaceae, genus Pyramidobacter were significantly higher than the Con-, however, the latter had members of the 


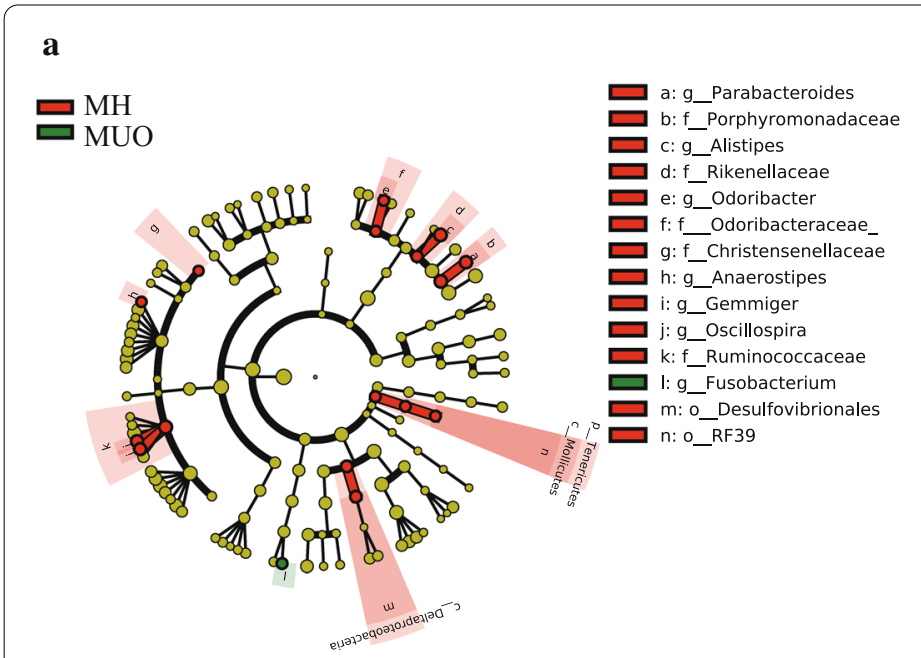

b

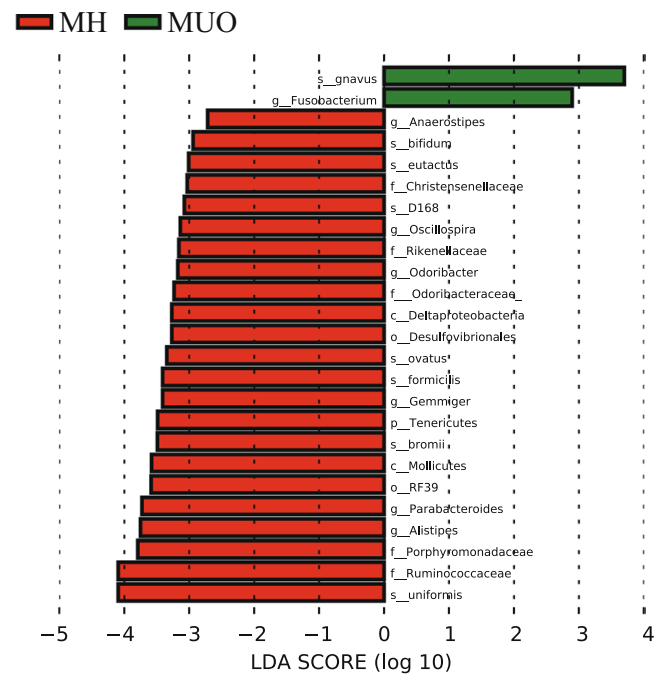

c

口 $\mathrm{MHO}$

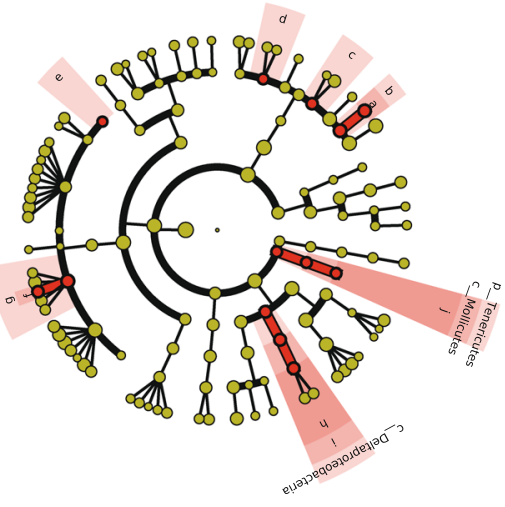

$\square$ a: g_Parabacteroides

b: f_Porphyromonadaceae

c: f_Rikenellaceae

d: f__odoribacteraceae

e:f Christensenellaceae

f: g_Gemmiger

g: f_Ruminococcaceae

$\square$ h: f_Desulfovibrionaceae

i: o Desulfovibrionales

j: o_RF39 d

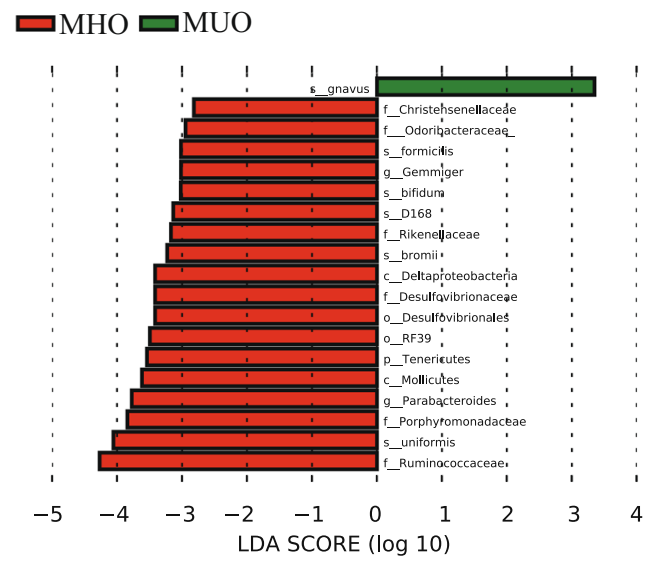

f e

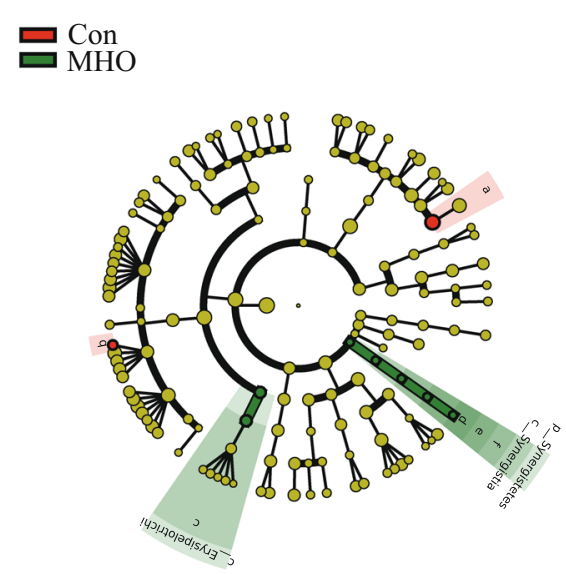

$\square$ a: f_Bacteroidaceae

b: g Anaerotruncus

c: o_Erysipelotrichales

d: g_Pyramidobacter

e:f_Dethiosulfovibrionaceae

f: o_synergistales
Con $\mathrm{MHO}$

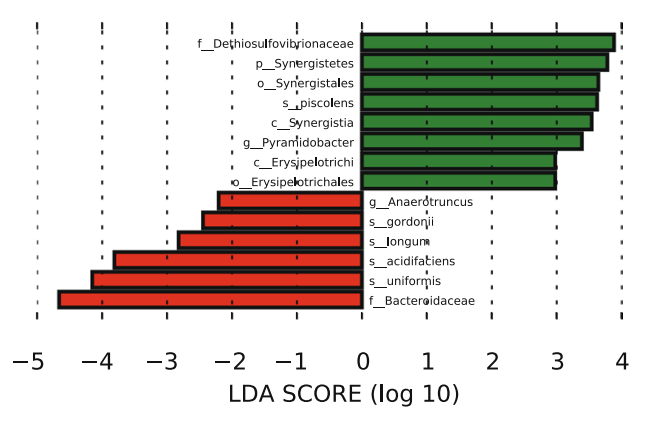

Fig. 3 Differential biomarkers associated with different metabolic status. A linear discriminant effect size (LeFse) analysis have been performed ( $a$ value $=0.05$, logarithmic LDA score threshold $=2.0$ ). MUO metabolic unhealthy obese, $M H O$ metabolically healthy obese, Con controls 
Table 3 The mean relative abundance of gut microbiota obese subjects with different metabolic status at phylum level

\begin{tabular}{lllll}
\hline & MHO & MUO & z & p value \\
\hline Actinobacteria & 0.012 & 0.025 & -0.783 & 0.434 \\
Bacteroidetes & 0.453 & 0.371 & -0.823 & 0.410 \\
Firmicutes & 0.393 & 0.321 & -0.919 & 0.358 \\
Fusobacteria & 0.006 & 0.016 & -1.494 & 0.135 \\
Proteobacteria & 0.132 & 0.267 & -0.535 & 0.593 \\
Tenericutes & 0.003 & 0.000 & -2.212 & 0.027 \\
Verrucomicrobia & 0.001 & 0.000 & -1.480 & 0.139 \\
\hline
\end{tabular}

MHO, metabolically healthy obese; $M U O$ : metabolic unhealthy obese

Italicized value $P<0.05$

Table 4 The mean relative abundance of gut microbiota with significantly differences in obese subjects with different metabolic status at genera level

\begin{tabular}{lllll}
\hline & MHO & Con & Z & P value \\
\hline Actinobacteria & 0.012 & 0.018 & -1.181 & 0.238 \\
Bacteroidetes & 0.319 & 0.377 & -1.006 & 0.314 \\
Cyanobacteria & 0.000 & 0.000 & -1.245 & 0.213 \\
Firmicutes & 0.572 & 0.531 & -0.831 & 0.406 \\
Fusobacteria & 0.006 & 0.014 & -0.324 & 0.746 \\
Proteobacteria & 0.088 & 0.057 & -1.881 & 0.060 \\
Synergistetes & 0.000 & 0.000 & -1.964 & 0.050 \\
Tenericutes & 0.002 & 0.002 & -1.408 & 0.159 \\
TM7 & 0.000 & 0.000 & -0.481 & 0.630 \\
Verrucomicrobia & 0.001 & 0.001 & -0.177 & 0.859 \\
\hline
\end{tabular}

MHO, metabolically healthy obese; Con, control

Italicized value $P<0.05$

family Bacteroidaceae, genus Anaerotruncus that were significantly higher (all p $<0.05$, Fig. 3e, f).

\section{Correlations between inflammatory factors and bacterial abundance}

To evaluate correlations between bacteria and serum inflammatory factors (IL-6, TNF- $\alpha$ and leptin), Spearman's rho cut-off values were assessed, taking into account $\mathrm{r}>0.4, \mathrm{r}<-0.4 \quad(\mathrm{p}<0.05$, Additional file 1 : Table S4).

For MUO subjects, Spearman's correlation analysis revealed that IL-6 positively correlated with genus Lactococcus, TNF- $\alpha$ positively correlated with phylum Bacteroidetes, negatively correlated with genus Citrobacter. LBP positively correlated with genus Prevotella, Odoribacter, and negatively correlated with genus Bifidobacterium, Streptococcus, Roseburia, Clostridium and Veillonella. Leptin positively correlated with genus
Eubacterium and negatively correlated with genus Faecalibacterium and Lachnospira (all $\mathrm{p}<0.05$, Additional file 1: Table S4).

For MHO subjects, Spearman's correlation analysis revealed that serum IL-6 positively correlated with genus Paraprevotella. LBP positively correlated with genus Roseburia and Faecalibacterium, and negatively correlated with genus Lactobacillus. Leptin positively correlated with phylum Bacteroidetes, Firmicutes, genus Phascolarctobacterium and negatively correlated with genus Dialister (all $\mathrm{p}<0.05$ ). There was no association between the bacteria and TNF $\alpha$ at the genus level (all $\mathrm{p}>0.05$ ).

\section{Metabolic pathway predictions}

A total of 15 KEGG pathways were generated using the composition of the fecal microbiota based on PICRUSt2 in the metabolic healthy cohorts (MHO and Con subjects) versus MUO subjects (Fig. 4, Additional file 1: Table S5). Importantly, the glucose metabolism pathways, including GDP-mannose biosynthesis and superpathway of UDP- $\mathrm{N}$-acetylglucosamine-derived $\mathrm{O}$-antigen building blocks biosynthesis, were increased in metabolic healthy cohorts and, conversely, the superpathway of fucose and rhamnose degradation were alternated in the metabolic healthy cohorts (all $\mathrm{p}<0.05)$. In the comparison between MHO and MUO subjects, we obtained 3 differential pathways including superpathway of fucose and rhamnose degradation, photorespiration, and sucrose degradation III, which were also observed significantly different between the metabolic healthy cohorts (MHO and Con subjects) versus MUO subjects (Fig. 4, Additional file 1: Table S6). Moreover, 11 differential metabolic patterns differentially expressed resulted in the comparison between $\mathrm{MHO}$ versus Con (Fig. 4, Additional file 1: Table S7).

\section{Discussion}

Recognized for decades, there is wide-ranging heterogeneity among obese individuals as to their risk for developing metabolic dysfunction and its attendant complications [12]. Also well-established, and which may contribute to this metabolic heterogeneity, is the fact those with central obesity are more prone to developing T2DM and cardiovascular disease than those with peripheral obesity [13]. In this study, to indirectly address the issue of fat distribution, we found there were no significant differences in WHR and WHtR between the two obese cohorts, MHO vs. MUO.

A chronic low-grade inflammation, triggered by nutrient surplus, is a constituent of obesity. Adipose-originated metabolic inflammation develops pari passu with insulin resistance and, as such, is a key element in the 
$\mathbf{a}$

$\square$ MHO\&Con $\square$ MUO

$95 \%$ confidence intervals

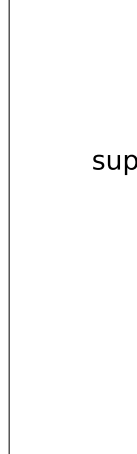

"UDP-2,3-diacetamido-2,3-dideoxy-\&alpha;-D-mannuro... formaldehyde assimilation I (serine pathway) |

superpathway of UDP-N-acetylglucosamine-derived O-... P colanic acid building blocks biosynthesis pyrimidine deoxyribonucleosides salvage

\section{photorespiration}

chondroitin sulfate degradation I (bacterial) $\square$

sucrose degradation III (sucrose invertase) uperpathway of fucose and rhamnose degradation superpathway of taurine degradation | superpathway of sulfolactate degradation superpathway of hexitol degradation (bacteria) mannan degradation

"1,4-dihydroxy-6-naphthoate biosynthesis I" P GDP-mannose biosynthesis

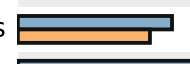

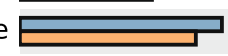

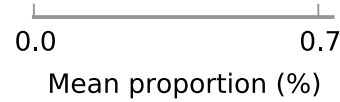

0.7
$1.67 e-3$

$9.29 \mathrm{e}-3$

0.014

0.018

0.024

0.027

0.031

0.032

0.033

0.037

0.041

0.042

0.044

0.044

0.046

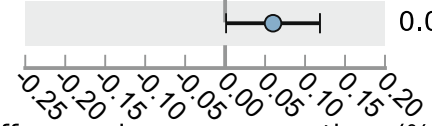

Difference in mean proportions (\%)

b

\section{$\square \mathrm{MHO} \square \mathrm{MUO}$} sucrose degradation III (sucrose invertase)
superpathway of fucose and rhamnose degradation

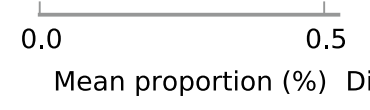

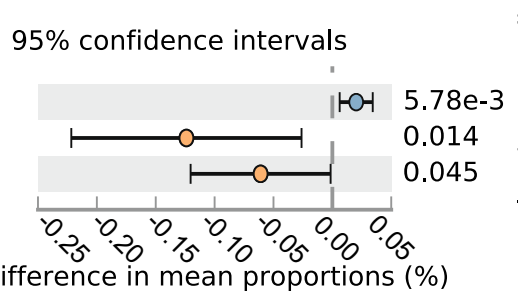

c

$\square$ Con $\square \mathrm{MHO}$

superpathway of pyridoxal 5'-phosphate biosynthesi...

dTDP-L-rhamnose biosynthesis I pyridoxal 5'-phosphate biosynthesis I chondroitin sulfate degradation I (bacterial) adenosine nucleotides degradation IV | superpathway of UDP-glucose-derived O-antigen buil... $\square$

"arginine, ornithine and proline interconversion" superpathway of adenosine nucleotides de novo bios... adenosine deoxyribonucleotides de novo biosynthesi... guanosine deoxyribonucleotides de novo biosynthesi... mycothiol biosynthesis

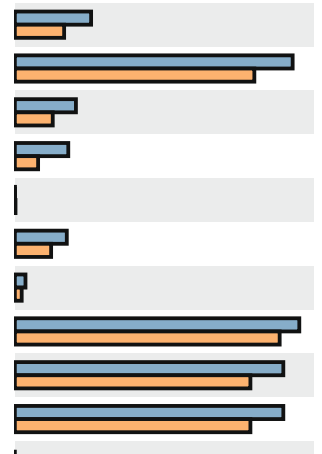

$95 \%$ confidence intervals

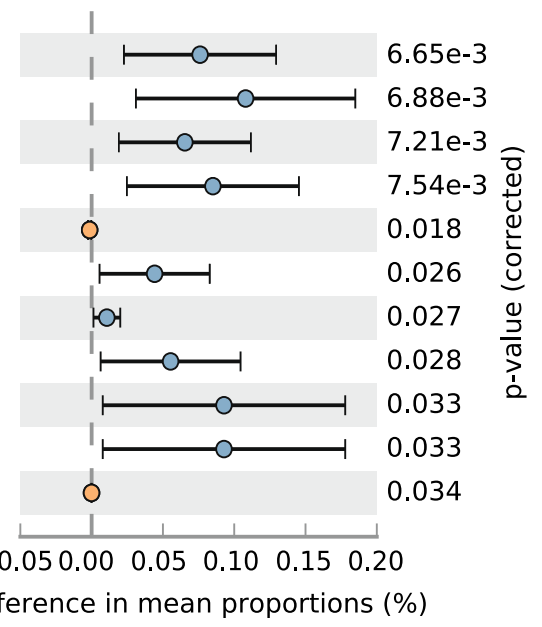

Fig. 4 KEGGs biomarkers associated with the three metabolic statuses. MUO metabolic unhealthy obese, MHO metabolically healthy obese, Con controls 
metabolic syndrome [14]. In this study, we found there were no significant differences in serum IL-6, TNF- $\alpha$, LBP and leptin between MHO and MUO subjects. It stands to reason that, besides these cytokines, other biochemical factors likely contribute to the metabolic diverseness in obese subjects. Or, perhaps, the concentrations of circulating compounds-such as those abovementioned-poorly reflect those found in extracellular or intracellular tissues.

Evidence can be adduced that the gut microbiota is involved in the aetiology of obesity and obesity-related complications such as nonalcoholic fatty liver disease, insulin resistance and T2DM $[15,16]$. These disorders are characterized by alterations in the diversity of the gut microbiota, and the relative abundance of certain genera. And bacteria-generated metabolites, translocated from the gut across a disrupted intestinal barrier, can affect several metabolic organs, such as the liver and adipose, thereby contributing to systemic metabolic inflammation [17].

Recently, several animal studies concluded that an optimal healthy-like gut microbiota may bestow a more propitious obese phenotype $[18,19]$. For instance, the abundance of Bacteroidetes and Tenericutes were closely aligned with bile acid metabolism and obesity-related inflammation in a murine model of the metabolic syndrome [20]. In our study, we corroborate this finding: reduced abundance of Tenericutes in the MUO group compared with the metabolically healthy groups (MHO and Con). Moreover, individuals with diminished insulin sensitivity had lower abundance of Tenericutes [21]. And, in animal experiments, administration of hydrogenated xanthohumol, which mitigates the metabolic syndrome by altering gut microbiota diversity and abundance, specifically, a reduction in Bacteroidetes and Tenericutes [20]. These results suggested an important role of Tenericutes in metabolism. We also observed greater abundance of Anaerostipes in the MHO and Con cohort, as well as the alpha and beta diversity. Using separatesample Mendelian randomization to obtain estimates of the associations of 27 genera of gut microbiota with cardiovascular disease risks, Anaerostipes was identified as being nominally associated with T2DM [22], and this effect may be a result of butyrate production [23]. These results buttress the notion of dysbiosis in the gut microbiota of MUO individuals.

To characterize the gut microbiota in obese children of different metabolic status, we further analyze the MHO and the MUO groups. The abundance of Tenericutes was significantly reduced in the MUO group compared with the metabolic healthy children, indicating that Tenericutes is related to the metabolic state, and the bacterial imbalance is independent of weight. Previously reported, the abundance of Parabacteroides was significantly decreased in obese subjects with metabolic syndrome [6], and nonalcoholic fatty liver disease [24], and negatively correlated with weight gain and leptin plasma levels [25]. And germane to our findings, both genera Gemmiger [26] and Parabacteroides [27] are gut bacteria negatively associated with obesity and disturbed host metabolism. In accordance, we found that that the fecal abundance of these bacteria was significantly higher in the MHO group compared with MUO.

The genera Parabacteroides are short-chain fatty acids (SCFAs)-producing bacteria. SCFAs are low molecular weight molecules produced from fermentation of dietary fiber or polysaccharides by gut microbiota. Absorbed by the intestinal epithelium into the blood, they can beget physiological disorders in the host, such as deranged lipid metabolism and intestinal environment imbalances [28, 29]. In our determination, alpha and beta diversity were significantly higher in Con and MHO children compared with the MUO group, again supporting the notion of dysbiosis in the unhealthy MUO population.

Notwithstanding that the gut microbiota of obese individuals with metabolic syndrome may indeed be unhealthy, is the gut microbiota of the MHO population really healthy? We compared the characteristic of gut microbiota in the Con and MHO children of different weights. Even though there was no significant difference in alpha and beta diversity, the relative abundances of phylum Synergistetes and genus Bacteroides were elevated in the MHO group compared to the Con children. Based on a metagenomic approach and bioinformatics analysis in obese adults, it is plausible that an abundance of the microbiota taxa Bacteroides could portent the evolution to T2DM [30].

Alterations in gut ecology can propel inflammatory pathways in several tissues, resulting in glucose intolerance and CVD [31, 32]. In rodents, a disturbance in the tripartite interactions between the microbiota, bile acids, and host metabolism, along with the bacterial production of lipopolysaccharides (LPS, i.e., endotoxemia), can beget derangements in glucose homeostasis [16, 26]. LBP is an acute inflammation phase protein that complexes with LPS and facilitates binding with CD14. In adolescents, serum LBP robustly correlates positively with indices of abnormal glucose and lipid metabolism. Herein, we found that, depending on the metabolic status, the serum levels of classic proinflammatory factors IL-6, TNF- $\alpha$, LBP and leptin were related to the abundance of various fecal bacteria. Notably, in MHO children, serum leptin correlated positively with genus Phascolarctobacterium and negatively with Dialister - the latter genera observed with low abundance in obese children [33]. And, relevant to our findings, it is noteworthy that Phascolarctobacterium is 
purportedly a biomarker for adult T2DM [30]. In high fat diet obese mice with insulin resistance, Prevotella was deemed as pro-inflammatory and, of note, its abundance in our study correlated with serum LBP [34]. As illustrated in our MHO children and the above-cited studies in humans, the gut microbiota is a marquee player in preserving normal metabolism despite obesity or, perhaps, an ephemeral protective microbiota destined to change with transition to MUO.

Compared to the metabolic healthy cohorts in the MUO children, several pathways associated with glucose and lipid metabolism pathways, such as fucose and rhamnose degradation and sucrose degradation III were increased. Conversely, mannan degradation was markedly decreased. Of interest, serum fucose levels are higher in the T2DM patients compared to healthy cohorts [35]. Mannan-oligosaccharide in the diet improves the metabolic syndrome in mice, alternatively insulin resistance and dyslipidemia [36, 37]. We found that bacterial fucose and rhamnose degradation and sucrose degradation III were increased in the MUO subjects compared with the $\mathrm{MHO}$ subjects, inferring that the change was independent of weight. However, insofar as serum levels of fucose were undetectable, and the dietary intake of sucrose and mannan were not assessed in our study, future longitudinal studies could conceivably unravel the intricate, possibly causual, relationships between the gut microbiota, obesity, and aberrant intermediary host metabolism.

\section{Conclusion}

In aggregate, the MUO population had lower alpha- and beta-diversity, and lower abundance of Tenericutes, inferring a robust intricate inter-relationship between gut bacterial ecology and host metabolic state. In the MHO population, phylum Synergistetes and genus Bacteroides and Phascolarctobacterium were more prevalent, and the abundance of some metabolism-related bacteria correlated with circulating proinflammatory factors, suggesting that compared to healthy controls, dysbiosis of gut microbiota was already extant in the MHO children, and conceivably a compensatory or remedial response to a surfeit of nutrients.

\section{Methods}

\section{Study population}

This study was approved by the Ethics Committee of the Fuzhou Children's Hospital of Fujian Medical University and, in all cases, informed consent was obtained.

The cross-sectional study consisted of participants managed by Fuzhou Children's Hospital of Fujian Medical University from September 2017 to March 2018. This study was limited to participants who met the following criteria: (a) ages between 5 to 15 years old, and (b) residence of Fujian province.
The exclusion criteria were as follows: any endocrine disorder, history of antibiotic therapy in the past 3 months prior to the enrollment, chronic gastrointestinal illness or use of gastro-intestinal-related medication, or diarrheal disease (World Health Organization definition) in the past 1 month.

\section{Clinical assessment}

Height and weight were measured by trained nurses. BMI-Z scores were calculated based on reference values of $\mathrm{Li}$ et al. [38]. At the end of normal expiration, waist and hip circumference were measured to the nearest $0.5 \mathrm{~cm}$ using standard technique with nonelastic tape. Waist circumference was measured at a point midway between the lower border of the ribs and the iliac crest, and hip circumference was measured at the widest part of the hip. A waist-to-hip ratio (WHR) was calculated by waist circumference $(\mathrm{cm})$ divided by hip circumference $(\mathrm{cm})$ and a waist-to-height ratio (WHtR) by waist circumference $(\mathrm{cm})$ divided by height $(\mathrm{cm})$.

\section{Laboratory methods}

All participants maintained their usual dietary pattern at least 3 days before blood sampling. After $12 \mathrm{~h}$ of fasting, $10 \mathrm{~mL}$ venous blood was drawn by registered nurses. All blood samples were stored at $-80{ }^{\circ} \mathrm{C}$, and analyzed within two weeks of sampling. Serum IL-6 was measured using a commercial ELISA kit (Abcam, UK), with an $4.4 \%$ inter-assay coefficient of variation (CV). Serum TNF- $\alpha$ levels was measured using a commercial ELISA kit (Abcam, UK), with inter-assay and intra-assay CVs of $3.3 \%$ and $9 \%$, respectively, and serum leptin assayed using a commercial ELISA kit (Abcam, UK), with interassay and intra-assay CVs of $2.4 \%$ and $2.7 \%$, respectively. The serum LBP levels were measured using a commercial ELISA kit (Abnova, Taiwan, China), with inter-assay and intra-assay CV 9.8-17.8\% and 6.1\%, respectively. Fasting plasma glucose (FPG) and plasma lipids, including total cholesterol (TC), triglyceride (TG), high-density lipoprotein cholesterol (HDL-c) and low density lipoprotein cholesterol (LDL-c), were assayed by standard methods using specific reagents (Beckman Coulter AU5800, USA). Fasting insulin (INS) was determined by a chemiluminescent immunoassay (IMMULITE 2000, Siemens Healthcare Diagnostics Products Limited, Germany). Fecal samples were collected and processed as previously described [39].

\section{Definition of metabolic unhealthy}

Metabolic syndrome parameters were applied according to 2019 Expert Committees [40], and MUO was 
defined by the presence of at least one of the following metabolic traits: (1) FPG $\geq 5.6 \mathrm{mmol} / \mathrm{L}$; (2) systolic blood pressure $\geq 90$ th percentile for gender and age; (3) fasting $\mathrm{HDL}-\mathrm{C}<1.03 \mathrm{mmol} / \mathrm{L}$; and (4) fasting TG $\geq 1.7 \mathrm{mmol} / \mathrm{L}$.

\section{Genomic DNA extraction and library construction}

The microbial community DNA was extracted and quantified as previously described [39]. Variable regions V3-V4 of bacterial 16s rRNA gene were amplified with degenerate PCR primers [39]. Libraries were qualified by the Agilent 2100 bioanalyzer (Agilent, USA). The validated libraries were used for sequencing on Illumina MiSeq platform (BGI, Shenzhen, China) following the standard pipeline of Illumina, and generating $2 \times 300 \mathrm{bp}$ paired-end reads.

\section{Statistical analysis}

Statistical analyses of clinical data were performed using the Statistical Package for the Social Sciences software version 23.0 (SPSS Inc. Chicago, IL, USA). The normality of the data was tested by Kolmogorov-Smirnov test. Data are expressed as mean \pm SD or median (25th-75th percentiles). Comparisons of the results were assessed using independent samples $\mathrm{t}$ test, Mann-Whitney $\mathrm{U}$ test and Kruskal-Wallis test, depending on the type of data distribution (e.g., non parametric). Comparison of rates between two groups was by chi-square. A value of $P<0.05$ was deemed statistically significant.

Statistical analysis of 16s rRNA sequencing data were performed on alpha- and beta-diversity measurements, which was done by software QIIME2 (v2019.7) [41]. Kruskal-Wallis Test was adopted for two groups comparison. Linear discriminant analysis Effect Size (LEfSe) Analysis was assessed by software LEFSE [42]. To predict metagenome functional content from 16S rRNA gene surveys, Picrust2 [43] have been applied to obtain the Kyoto Encyclopedia of Genes and Genomes (KEGG) pathways, and STAMP [44] was used to analyze the differential pathways.

\section{Supplementary Information}

The online version contains supplementary material available at https://doi. org/10.1186/s12934-021-01548-9.

Additional file 1: Table $\mathbf{S 1}$. The mean relative abundance of gut microbiota in different metabolic status at phylum level. Table S2. Comparison of alpha-diversity in obese subjects with different metabolic status. Table S3. Comparison of beta-diversity between different metabolic status. Table S4. Spearman's correlation table on OTUs and inflammatory factors in MHO and MUO groups. Table S5. KEGGs biomarkers in $\mathrm{MHO}$ and Con subjects compared with MUO subjects. Table S6. KEGGs biomarkers in $\mathrm{MHO}$ and MUO subjects. Table S7. KEGGs biomarkers in $\mathrm{MHO}$ and Con subjects.
Acknowledgements

The authors are grateful to all the participants.

\section{Authors' contributions}

XY drafted the initial manuscript; RMC conceptualized and designed the study, and reviewed and revised the manuscript; KLM assisted in data analysis and manuscript composition; YZ and XHY collected cases; XQL did the laboratory testing. All authors read and approved the final manuscript.

\section{Funding}

This study was supported by Technology Innovation Team Train Project of Fuzhou Health Committee in China (2016-S-wp1), and sponsored by key Clinical Specialty Discipline Construction Program of Fuzhou, Fujian, P.R.C. (201610191) and Fuzhou Children's Medical Center (2018080310).

\section{Availability of data and materials}

The original contributions presented in the study are publicly available. The raw sequence data reported in this paper have been deposited in the Genome Sequence Archive (Genomics, Proteomics \& Bioinformatics 2017) in National Genomics Data Center (Nucleic Acids Res 2020), Beijing Institute of Genomics (China National Center for Bioinformation), Chinese Academy of Sciences, under accession number CRA003010 that are publicly accessible at https://bigd.big.ac.cn/gsa.

\section{Ethics approval and consent to participate}

This study was reviewed and approved by the Ethics Committee of Fuzhou Children's Hospital of Fujian Medical University, and was conducted in agreement with the Declaration of Helsinki Principles. Informed consent was obtained from all individual participants included in the study.

\section{Consent for publication}

Informed consent for publication was obtained from all individual participants included in the study.

\section{Competing interests}

The authors declare that they have no competing interests.

\section{Author details}

${ }^{1}$ Department of Endocrinology, Fuzhou Children's Hospital of Fujian Medical University, NO. 145, 817 Middle Road, Fuzhou 350005, China. ${ }^{2}$ Division of Pediatric Endocrinology and Diabetes, University of Alabama at Birmingham, Birmingham, AL 35233, USA.

Received: 8 December 2020 Accepted: 18 February 2021

Published online: 27 February 2021

References

1. The CR, Picture B. Outrunning child obesity trends. BMJ. 2018;363:k4362. https://doi.org/10.1136/bmj.k4362.

2. Damanhoury S, Newton AS, Rashid M, Hartling L, Byrne JLS, Ball GDC. Defining metabolically healthy obesity in children: a scoping review. Obes Rev. 2018;19(11):1476-91. https://doi.org/10.1111/obr.12721.

3. Greenhill C. Redefining metabolically healthy obesity. Nat Rev Endocrinol. 2018;14(8):440. https://doi.org/10.1038/s41574-018-0046-9.

4. Garcia-Moll X. Obesity and prognosis: time to forget about metabolically healthy obesity. Eur Heart J. 2018;39(5):407-9. https://doi.org/10.1093/ eurheartj/ehx535.

5. Gérard P. Gut microbiota and obesity. Cell Mol Life Sci. 2016;73(1):147-62. https://doi.org/10.1007/s00018-015-2061-5.

6. Gallardo-Becerra L, Cornejo-Granados F, García-López R, Valdez-Lara A, Bikel S, Canizales-Quinteros S, López-Contreras BE, Mendoza-Vargas A, Nielsen H, Ochoa-Leyva A. Metatranscriptomic analysis to define the Secrebiome, and 16S rRNA profiling of the gut microbiome in obesity and metabolic syndrome of Mexican children. Microb Cell Fact. 2020;19(1):61. https://doi.org/10.1186/s12934-020-01319-y.

7. Ma Q, Li Y, Li P, Wang M, Wang J, Tang Z, Wang T, Luo L, Wang C, Wang T, Zhao B. Research progress in the relationship between type 2 diabetes mellitus and intestinal flora. Biomed Pharmacother. 2019;117:109138. https://doi.org/10.1016/j.biopha.2019.109138. 
8. Rial SA, Karelis AD, Bergeron KF, Mounier C. Gut microbiota and metabolic health: the potential beneficial effects of a medium chain triglyceride diet in obese individuals. Nutrients. 2016;8(5):281. https://doi.org/10.3390/ nu8050281.

9. Iacobini C, Pugliese G, Blasetti Fantauzzi C, Federici M, Menini S. Metabolically healthy versus metabolically unhealthy obesity. Metabolism. 2019;92:51-60. https://doi.org/10.1016/.j.metabol.2018.11.009.

10. Yuan $X$, Chen R, Ouyang $Q$, Lin X, Ai Z, Zhang Y, Yang X. Novel associations of serum adropin and lipopolysaccharide-binding protein versus lipid profiles in childhood obesity. J Pediatr Endocrinol Metab. 2020;33(2):26570. https://doi.org/10.1515/jpem-2019-0329.

11. Nirmalkar K, Murugesan S, Pizano-Zárate ML, Villalobos-Flores LE, GarcíaGonzález C, Morales-Hernández RM, Nuñez-Hernández JA, HernándezQuiroz F, Romero-Figueroa MDS, Hernández-Guerrero C, Hoyo-Vadillo C, García-Mena J. Gut microbiota and endothelial dysfunction markers in obese mexican children and adolescents. Nutrients. 2018;10(12):2009. https://doi.org/10.3390/nu10122009.

12. Viveiros A, Oudit GY. The dual nature of obesity in metabolic programming: quantity versus quality of adipose tissue. Clin Sci. 2020;134(18):2447-51. https://doi.org/10.1042/CS20201028.

13. Brandão I, Martins MJ, Monteiro R. Metabolically healthy obesityheterogeneity in definitions and unconventional factors. Metabolites. 2020;10(2):E48. https://doi.org/10.3390/metabo10020048.

14. Kaciríová M, Zmeškalová A, Kořinková L, Železná B, Kuneš J, Maletínská L. Inflammation: major denominator of obesity, type 2 diabetes and Alzheimer's disease-like pathology? Clin Sci. 2020;134(5):547-70. https:// doi.org/10.1042/CS20191313.

15. Canfora EE, Meex RCR, Venema K, Blaak EE. Gut microbial metabolites in obesity, NAFLD and T2DM. Nat Rev Endocrinol. 2019;15(5):261-73. https ://doi.org/10.1038/s41574-019-0156-Z.

16. Pedersen HK, Gudmundsdottir $V$, Nielsen HB, Hyotylainen T, Nielsen T, Jensen BA, Forslund K, Hildebrand F, Prifti E, Falony G, Le Chatelier E, Levenez F, Doré J, Mattila I, Plichta DR, Pöhö P, Hellgren LI, Arumugam M, Sunagawa S, Vieira-Silva S, Jørgensen T, Holm JB, Trošt K, MetaHIT Consortium, Kristiansen K, Brix S, Raes J, Wang J, Hansen T, Bork P, Brunak $\mathrm{S}$, Oresic M, Ehrlich SD, Pedersen O. Human gut microbes impact host serum metabolome and insulin sensitivity. Nature. 2016;535(7612):37681. https://doi.org/10.1038/nature18646.

17. Tilg $H, Z$ mora N, Adolph TE, Elinav E. The intestinal microbiota fuelling metabolic inflammation. Nat Rev Immunol. 2020;20(1):40-54. https://doi. org/10.1038/s41577-019-0198-4.

18. Serino M, Luche E, Gres S, Baylac A, Bergé M, Cenac C, Waget A, Klopp P, lacovoni J, Klopp C, Mariette J, Bouchez O, Lluch J, Ouarné F, Monsan $P$, Valet P, Roques C, Amar J, Bouloumié A, Théodorou V, Burcelin R. Metabolic adaptation to a high-fat diet is associated with a change in the gut microbiota. Gut. 2012;61(4):543-53. https://doi.org/10.1136/gutjn 1-2011-301012.

19. Neyrinck AM, Van Hée VF, Piront N, De Backer F, Toussaint O, Cani PD, Delzenne NM. Wheat-derived arabinoxylan oligosaccharides with prebiotic effect increase satietogenic gut peptides and reduce metabolic endotoxemia in diet-induced obese mice. Nutr Diabetes. 2012;2(1):e28. https://doi.org/10.1038/nutd.2011.24.

20. Zhang Y, Bobe G, Revel JS, Rodrigues RR, Sharpton TJ, Fantacone ML, Raslan K, Miranda CL, Lowry MB, Blakemore PR, Morgun A, Shulzhenko N, Maier CS, Stevens JF, Gombart AF. Improvements in metabolic syndrome by xanthohumol derivatives are linked to altered gut microbiota and bile acid metabolism. Mol Nutr Food Res. 2020;64(1):e1900789. https://doi. org/10.1002/mnfr.201900789.

21. Benedict C, Vogel H, Jonas W, Woting A, Blaut M, Schürmann A, Cedernaes J. Gut microbiota and glucometabolic alterations in response to recurrent partial sleep deprivation in normal-weight young individuals. Mol Metab. 2016;5(12):1175-86. https://doi.org/10.1016/j.molme t.2016.10.003.

22. Yang Q, Lin SL, Kwok MK, Leung GM, Schooling CM. The roles of 27 genera of human gut microbiota in ischemic heart disease, type 2 diabetes mellitus, and their risk factors: a mendelian randomization study. Am J Epidemiol. 2018;187(9):1916-22. https://doi.org/10.1093/aje/kwy096.

23. Koh A, De Vadder F, Kovatcheva-Datchary P, Bäckhed F. From dietary fiber to host physiology: short-chain fatty acids as key bacterial metabolites. Cell. 2016;165(6):1332-45. https://doi.org/10.1016/j.cell.2016.05.041.
24. Monga Kravetz A, Testerman T, Galuppo B, Graf J, Pierpont B, Siebel S, Feinn R, Santoro N. Effect of gut microbiota and PNPLA3 rs738409 variant on nonalcoholic fatty liver disease (NAFLD) in obese youth. J Clin Endocrinol Metab. 2020;105(10):dgaa382. https://doi.org/10.1210/cline $\mathrm{m} / \mathrm{dgaa3} 32$.

25. Carbajo-Pescador S, Porras D, García-Mediavilla MV, Martínez-Flórez S, Juarez-Fernández M, Cuevas MJ, Mauriz JL, González-Gallego J, Nistal E, Sánchez-Campos S. Beneficial effects of exercise on gut microbiota functionality and barrier integrity, and gut-liver crosstalk in an in vivo model of early obesity and non-alcoholic fatty liver disease. Dis Model Mech. 2019;12(5):dmm039206. https://doi.org/10.1242/dmm.039206.

26. Brown JM, Hazen SL. Microbial modulation of cardiovascular disease. Nat Rev Microbiol. 2018;16:171-81. https://doi.org/10.1038/nrmicro.2017.

27. Patrone V, Vajana E, Minuti A, Callegari ML, Federico A, Loguercio C, Dallio $\mathrm{M}$, Tolone S, Docimo L, Morelli L. Postoperative changes in fecal bacterial communities and fermentation products in obese patients undergoing bilio-intestinal bypass. Front Microbiol. 2016;7:200. https://doi. org/10.3389/fmicb.2016.00200.

28. Jing N, Liu X, Jin M, Yang X, Hu X, Li C, Zhao K. Fubrick tea attenuates high-fat diet induced fat deposition and metabolic disorder by regulating gut microbiota and caffeine metabolism. Food Funct. 2020;11(8):697186. https://doi.org/10.1039/d0fo01282c.

29. Hold GL. The gut microbiota, dietary extremes and exercise. Gut. 2014;63(12):1838-9. https://doi.org/10.1136/gutjnl-2014-307305.

30. Wang TY, Zhang XQ, Chen AL, Zhang J, Lv BH, Ma MH, Lian J, Wu YX, Zhou YT, Ma CC, Dong RJ, Ge DY, Gao SH, Jiang GJ. A comparative study of microbial community and functions of type 2 diabetes mellitus patients with obesity and healthy people. Appl Microbiol Biotechnol. 2020;104(16):7143-53. https://doi.org/10.1007/s00253-020-10689-7.

31. Biassoni R, Di Marco E, Squillario M, Barla A, Piccolo G, Ugolotti E, Gatti C, Minuto N, Patti G, Maghnie M, d'Annunzio G. Gut microbiota in T1DMonset pediatric patients: machine-learning algorithms to classify microorganisms as disease linked. J Clin Endocrinol Metab. 2020;105(9):dgaa407. https://doi.org/10.1210/clinem/dgaa407.

32. van de Guchte M, Blottière HM, Doré J. Humans as holobionts: implications for prevention and therapy. Microbiome. 2018;6:81. https://doi. org/10.1186/s40168-018-0466-8.

33. Wahlström A, Sayin SI, Marschall HU, Bäckhed F. Intestinal crosstalk between bile acids and microbiota and its impact on host metabolism. Cell Metab. 2016;24(1):41-50. https://doi.org/10.1016/j.cmet.2016.05.005.

34. Cai W, Xu J, Li G, Liu T, Guo X, Wang H, Luo L. Ethanol extract of propolis prevents high-fat diet-induced insulin resistance and obesity in association with modulation of gut microbiota in mice. Food Res Int. 2020;130:108939. https://doi.org/10.1016/j.foodres.2019.108939.

35. Al-Aama JY, Al Mahdi HB, Salama MA, Bakur KH, Alhozali A, Mosli HH, Bahijri SM, Bahieldin A, Willmitzer L, Edris S. Detection of secondary metabolites as biomarkers for the early diagnosis and prevention of type 2 diabetes. Diabetes Metab Syndr Obes. 2019;17(12):2675-84. https://doi. org/10.2147/DMSO.S215528.

36. Wang H, Zhang X, Wang S, Li H, Lu Z, Shi J, Xu Z. Mannan-oligosaccharide modulates the obesity and gut microbiota in high-fat diet-fed mice. Food Funct. 2018;9(7):3916-29. https://doi.org/10.1039/c8fo00209f.

37. Chen H, Nie Q, Hu J, Huang X, Zhang K, Pan S, Nie S. Hypoglycemic and hypolipidemic effects of glucomannan extracted from Konjac on type 2 diabetic rats. J Agric Food Chem. 2019;67(18):5278-88. https://doi. org/10.1021/acs.jafc.9b01192.

38. Li H, Zong XN, Ji CY, Mi J. Body mass index cut-offs for overweight and obesity in Chinese children and adolescents aged 2-18 years. Zhonghua Liu Xing Bing Xue Za Zhi. 2010;31(6):616-20.

39. Yuan $X$, Chen $R$, Zhang $Y$, Lin $X$, Yang $X$. Sexual dimorphism of gut microbiota at different pubertal status. Microb Cell Fact. 2020;19(1):152. https:// doi.org/10.1186/s12934-020-01412-2.

40. Liu C, Wang C, Guan S, Liu H, Wu X, Zhang Z, Gu X, Zhang Y, Zhao Y, Tse LA, Fang $X$. The prevalence of metabolically healthy and unhealthy obesity according to different criteria. Obes Facts. 2019;12(1):78-90. https ://doi.org/10.1159/000495852.

41. Bolyen E, Rideout JR, Dillon MR, Bokulich NA, Abnet CC, Al-Ghalith GA, Alexander H, Alm EJ, Arumugam M, Asnicar F, Bai Y, Bisanz JE, Bittinger K, Brejnrod A, Brislawn CJ, Brown CT, Callahan BJ, Caraballo-Rodríguez AM, Chase J, Cope EK, Da Silva R, Diener C, Dorrestein PC, Douglas GM, Durall DM, Duvallet C, Edwardson CF, Ernst M, Estaki M, Fouquier J, Gauglitz JM, 
Gibbons SM, Gibson DL, Gonzalez A, Gorlick K, Guo J, Hillmann B, Holmes S, Holste H, Huttenhower C, Huttley GA, Janssen S, Jarmusch AK, Jiang L, Kaehler BD, Kang KB, Keefe CR, Keim P, Kelley ST, Knights D, Koester I, KosciolekT, Kreps J, Langille MGI, Lee J, Ley R, Liu YX, Loftfield E, Lozupone C, Maher M, Marotz C, Martin BD, McDonald D, Mclver LJ, Melnik AV, Metcalf JL, Morgan SC, Morton JT, Naimey AT, Navas-Molina JA, Nothias LF, Orchanian SB, Pearson T, Peoples SL, Petras D, Preuss ML, Pruesse E, Rasmussen LB, Rivers A, Robeson MS 2nd, Rosenthal P, Segata N, Shaffer M, Shiffer A, Sinha R, Song SJ, Spear JR, Swafford AD, Thompson LR, Torres PJ, Trinh P, Tripathi A, Turnbaugh PJ, UI-Hasan S, van der Hooft JJJ, Vargas F, Vázquez-Baeza Y, Vogtmann E, von Hippel M, Walters W, Wan Y, Wang M, Warren J, Weber KC, Williamson CHD, Willis AD, Xu ZZ, Zaneveld JR, Zhang Y, Zhu Q, Knight R, Caporaso JG. Reproducible, interactive, scalable and extensible microbiome data science using QIIME 2. Nat Biotechnol. 2019;37(8):852-7. https://doi.org/10.1038/s41587-019-0209-9.
42. Segata N, Izard J, Waldron L, Gevers D, Miropolsky L, Garrett WS, Huttenhower $C$. Metagenomic biomarker discovery and explanation. Genome Biol. 2011;12(6):R60. https://doi.org/10.1186/gb-2011-12-6-r60.

43. Douglas GM, Maffei VJ, Zaneveld JR, Yurgel SN, Brown JR, Taylor CM, Huttenhower C, Langille MGI. PICRUSt2 for prediction of metagenome functions. Nat Biotechnol. 2020;38(6):685-8. https://doi.org/10.1038/ s41587-020-0548-6.

44. Parks DH, Tyson GW, Hugenholtz P, Beiko RG. STAMP: statistical analysis of taxonomic and functional profiles. Bioinformatics. 2014;30(21):3123-4. https://doi.org/10.1093/bioinformatics/btu494.

\section{Publisher's Note}

Springer Nature remains neutral with regard to jurisdictional claims in published maps and institutional affiliations.
Ready to submit your research? Choose BMC and benefit from:

- fast, convenient online submission

- thorough peer review by experienced researchers in your field

- rapid publication on acceptance

- support for research data, including large and complex data types

- gold Open Access which fosters wider collaboration and increased citations

- maximum visibility for your research: over 100M website views per year

At BMC, research is always in progress.

Learn more biomedcentral.com/submissions 